\title{
RAS Family Gene Mutation
}

National Cancer Institute

\section{Source}

National Cancer Institute. RAS Family Gene Mutation. NCI Thesaurus. Code C132229.

A change in the nucleotide sequence in a RAS family gene (HRAS, KRAS or NRAS). 